Cinémas

Revue d'études cinématographiques

Journal of Film Studies

GARDIES, André. L'Espace au cinéma. Paris : Méridiens

Klincksieck, 1993, 222 p.

GARDIES, André. Le Récit filmique. Paris: Hachette, 1993, 155 p.

\title{
Pierre Grégoire
}

Volume 5, numéro 1-2, automne 1994

URI : https://id.erudit.org/iderudit/1001016ar

DOI : https://doi.org/10.7202/1001016ar

Aller au sommaire du numéro

Éditeur(s)

Cinémas

ISSN

1181-6945 (imprimé)

1705-6500 (numérique)

Découvrir la revue

Citer ce compte rendu

Grégoire, P. (1994). Compte rendu de [GARDIES, André. L'Espace au cinéma.

Paris : Méridiens Klincksieck, 1993, 222 p. / GARDIES, André. Le Récit filmique.

Paris : Hachette, 1993, 155 p.] Cinémas, 5(1-2), 229-232.

https://doi.org/10.7202/1001016ar 
GARDIES, André. L'Espace au cinéma. Paris : Méridiens Klincksieck, 1993, 222 p. Le Récit filmique. Paris: Hachette, 1993, $155 \mathrm{p}$.

Entrepris dès son ouvrage consacré au cinéma africain, dont le sous-titre était "l'espace-miroir ${ }^{1}$ ", poursuivi à travers différents articles du lexique théorique qu'il a signé avec Jean Bessa${ }^{l e l}{ }^{2}$, le travail d'André Gardies sur l'espace débouche enfin sur un livre, L'Espace au cinéma, qui a tout d'une somme. Posé comme constituant fondamental du spectacle cinématographique, l'espace se trouve étudié non pas sous son seul aspect physique ou plastique, mais plutôt à la croisée de l'abstrait et du concret, "dans sa dimension structurelle et systématique" (p. 12). Se situant dans la perspective sémio-narratologique, Gardies nous présente le film narratif comme un lieu où s'emboîtent et s'articulent quatre différents espaces, chacun faisant l'objet d'une partie du livre: l'espace cinématographique, l'espace diégétique, l'espace narratif, l'espace du spectateur.

L'« espace cinématographique ", j'y accède dès l'instant où, ayant choisi un siège dans la salle, je prends place au sein de l'institution cinématographique, instigatrice bientôt de la métamorphose qui fera de moi, sujet social inscrit dans l'ordre du quotidien, un autre être: le sujet "spectaculaire", tout heureux de se plier aux prescriptions du "dispositif" qui lui livrera son plaisir. Parmi ces dernières, outre les conditions de réception (état de "sous-motricité et de sur-perception" [Metz], obscurité, élévation et démesure de l'écran, etc.), il y a celles qui assurent "l'émergence du filmique" (p. 39), soit les fameuses "matières de l'expression " que Gardies propose de distinguer non plus d'après leur nature, mais selon leur mode de signification, réduisant leur nombre à trois : l'iconique (englobant l'image et le brui- 
tage) "qui figure l'espace tout en le structurant", le verbal qui "énonce" l'espace, et le musical qui le connote (p. 39-55). Une fois assumées les contraintes de l'espace institutionnel, je puis entrer en fiction. C'est dans la seconde partie du livre, "L'espace diégétique", que l'auteur développe un des éléments les plus dynamiques et les plus féconds de sa théorie: cette distinction qu'il opère entre l'espace et le lieu. Reprenant la célèbre dyade de Saussure, Gardies postule que le lieu est à l'espace ce que la parole est à la langue - le lieu, "parole de l'espace", écrit-il (p. 86). Le lieu "actualise" ou "figure" l'espace, qui est un système abstrait, il lui donne une réalité qu'on peut voir. L'espace découle du "cognitif", le lieu relève du "perceptible" (p. 90); on conçoit l'espace du western, mais ce qu'on regarde palpiter dans la diégèse, ce sont ses lieux: le saloon, la banque, la prison. À travers de convaincantes analyses, portant notamment sur Le Salaire de la peur et Shining, l'auteur nous montre que l'espace diégétique constitue une construction, à laquelle travaille, bien sûr, le film en figurant les lieux, mais à laquelle aussi travaille activement le spectateur, en mêlant à son regard un savoir extérieur au film - ces «lieux communs" que j'ai en tête.

Glissant de Saussure à Greimas, Gardies présente, dans la troisième partie de son ouvrage — "L'espace narratif" l'espace-lieu comme l'un des principaux "actants" du récit filmique. $\mathrm{Ce}$ "partenaire" à part entière de la narrativité, l'auteur le situe à égalité du personnage (dit "sujet") avec qui l'espace entretient un échange constant de "valeurs", et qui constitue la dynamique même du récit. Dans cette perspective, "ce qui fonde le réseau des déplacements du héros, c'est moins le déterminisme de la consécution que la nécessité de se rendre en des lieux spécifiques pour y acquérir les valeurs dont il a besoin pour sa quête" (p. 149). Toujours à la lumière du Salaire de la peur, et affirmant que tout récit raconte, au fond, l'histoire d'un sujet dans ses rapports à un espace, Gardies définit "l'effet "Belle au bois dormant" " (p. 131-136): le Prince charmant, de retour, éveille la fonctionnalité narrative du palais endormi, qui en échange collabore à son propre développement comme personnage. La fonction actantielle de l'espace se trouve en outre représentée par les "opérations élémentaires" ou "récits minimaux" 
auxquels se résume toute histoire, et que l'auteur définit en termes de relations de conjonction et de disjonction: par exemple, le récit typique de l'erreur judiciaire se traduit par une séquence où le sujet se trouve d'abord disjoint de l'espace (il est libre), puis conjoint à lui (il est mis en prison) pour enfin retrouver la disjonction de départ (p. 150-154). Dans la quatrième partie, "L'espace du spectateur", Gardies rappelle que le récit construit, en même temps que sa fable, sa stratégie d'adresse, son mode d'énonciation - cet "espace" purement communicationnel du fait filmique - ainsi qu'un espace propre à l'activité du spectateur. Trois différents espaces peuplent l'espace spectatoriel: 1) l'espace textuel, soit le champ de l'histoire, que le spectateur parcourt en tous sens, avec ses yeux, sa mémoire, son savoir; 2) l'espace de l'énonciation, où s'accomplit, dans cet "acte illocutoire" que constitue "la situation d'énonciation filmique" (p. 180), la "rencontre" du film et du spectateur, et où ce dernier perçoit ici et là, à l'œuvre dans le déroulement du texte, des stratégies visant à le capter, quelque chose comme l'affleurement, dans le tissu textuel, d'une personne-origine, ou le souffle d'une "voix" ; 3) l'espace du savoir spectatoriel. L'auteur rappelle ici, sans y ajouter grand-chose, l'essentiel des théories des dernières années sur la focalisation, proposant d'adjoindre à cette dernière la notion de polarisation qui "renvoie non seulement au réglage du savoir mais aussi à sa conséquence : le "positionnement" narratif du spectateur" (p. 202).

On peut regretter que L'Espace au cinéma, qui à ce jour constitue assurément le texte majeur de son auteur, se termine sur son chapitre sans doute le moins novateur. Y affleure, à nu, peut-être le seul défaut du livre, cette tendance excessive à la classification et à l'encyclopédisme, qui alourdit le texte de ce théoricien en revanche armé d'une langue remarquablement claire et, notamment, d'une rigueur lexicale qui font hélas défaut à bien des théoriciens actuels.

Publié la même année, Le Récit filmique, pour sa part, apparaît comme une version remaniée et abrégée, à des fins scolaires, de l'ouvrage précédent, "polarisée " autour du problème de la spécificité du narratif au cinéma. Livre en trois parties enrichi d'extraits de textes clés (Souriau, Barthes, Metz, Eco, etc.) et d'un 
glossaire, il rappelle, dans le premier temps, "les conditions cinématographiques du récit filmique" (le dispositif institutionnel, les matières de l'expression), les principes de son fonctionnement (la nature de la "narrativité", définie comme "un ensemble de codes, de procédures et d'opérations [...] dont la présence dans un texte permet de reconnaître ce dernier comme étant un récit ", p. 28) et de sa lecture (le "contrat" de la fiction). La seconde partie définit les trois principales composantes du monde diégétique, soit le personnage, l'espace, le temps. L'auteur étend ici à la "figure actorielle" (agrégat de l'actant, du rôle, du personnage et du comédien, p. 59-63) le caractère actantiel qu'il attribue à l'espace; dans le chapitre consacré au temps, il s'en tient essentiellement à résumer les travaux de Genette sur la double temporalité (temps de l'histoire, temps du récit) et sur les trois aspects du temps (ordre, durée, fréquence). La troisième partie étudie les stratégies d'énonciation qu'emploie le récit filmique pour régler la participation du spectateur. Gardies y précise notamment la notion de localisation en rapport avec la monstration: la première, physique et optique, dépend de la place occupée par la caméra et est liée à la formation de l'image, alors que la seconde procède des stratégies narratives — la localisation construit l'œil spectatoriel; la monstration, elle, le transforme en regard (p. 100-106). L'auteur conclut qu' "en fait, c'est dans sa façon de “dialoguer" avec le spectateur que le récit filmique manifeste sa plus grande singularité" (p. 134).

Par les intuitions brillantes qu'ils contiennent, par le courant disons poétique qui parfois les traverse (le dyade espace-lieu, et, dans le second livre, cette interrogation de la "voix" de l'énonciation), ces deux ouvrages laissent rêver d'un suivant, où un André Gardies dégagé de tout didactisme explorerait plus librement les "espaces" théoriques qu'il sait si bien susciter.

Pierre Grégoire

Université de Montréal

\section{NOTES}

1 Cinéma d'Afrique noire francoPhone; l'espace-miroir. Paris: L'Harmattan, 1989.

2200 mots clés de la théorie du cinéma. Paris: Cerf, 1992. 


\section{Ouvrages reçus / Books Received}

CAUTE, David. Joseph Losey. A Revenge on Life. New York: Oxford University Press, 1994, 591 p.

FALSETTO, Mario. Stanley Kubrick. A Narrative and Stylistic Analysis. Westport/London, Praeger, 1994, 217 p.

JOST, François. Un monde à notre image. Énonciation, cinéma, télévision. Paris : Klincksieck, 1992, 140 p.

LABERGE, Yves (direction). "À l'affiche: cent ans de cinéma au Québec", Cap-aux-Diamants, nº 38 (été 1994) 70 p.

MÜLlER, Jürgen E. (dir.). Towards a Pragmatics of the Audiovisual. Theory and History. Volume 1. Münster : Nodus Publikationen, 1994, 199 p.

NASTA, Dominique, AUBENAS, Jacqueline. "Toto le Héros. Itinéraires d'une première œuvre", Revue belge du cinéma, $\mathrm{n}^{\text {os }}$ 36-37 (1994) $96 \mathrm{p}$.

PELLETIER, Esther, RAYNAULD, Isabelle (direction). "Le Scénario de film ", Études littéraires, vol. 26, n 2 (automne 1993) $132 \mathrm{p}$.

SNOW, Michael. The Michael Snow Project. The Collected Writings of Michael Snow. With a foreword by Louise Dompierre. Waterloo: Wilfrid Laurier University Press, 1994, $295 \mathrm{p}$. 